\title{
Object-Oriented Fuzzy Database Representation through UML
}

\author{
Santosh Kumar, PhD \\ Head and Assistant Professor \\ Department of Computer Science \& Engineering \\ Maharishi University of Information Technology, \\ Lucknow
}

\author{
Shweta Dwivedi \\ Research Scholar \\ Department of Computer Science \& Engineering \\ Maharishi University of Information Technology, \\ Lucknow
}

\begin{abstract}
Due to the rapid use of various applications, the information is collected from these applications are vague and improper but the crisp information is an important part of any objectoriented database in the current scenario. As an uncertainty orvagueness in any database is represented through the fuzzy techniques, an object-oriented database is very efficient in handling that kind of fuzziness and complex database model. Therefore, the limited research work has done to designing and representing a fuzzy object-oriented database through UML, for that purpose, a domain of hospital has been taken for diagnosis the patient for various diseases with their uncertain input values (disease sign, symptoms, and pathology test result values). This will enhance the functioning of the developed software design on the present approach. A UML class \& Sequence diagram are also designed for representing the complete process of registration and diagnosis of patients and design the fuzzy object-oriented database i.e. FOOD through the SQL server 2008.
\end{abstract}

\section{Keywords}

UML, Object-Oriented Database, Fuzzy Object-Oriented Database (FOOD), Class Diagram and Sequence Diagram.

\section{INTRODUCTION}

Graphical representation is one of the important parts of any object-oriented problem. A well-known modeling language i.e. Unified Modeling Language (UML) is widely used in every field of world for solving any object-oriented problem. UML is very rich in nations for designing and deploying any real world problem in diagrammatic form. In the current scenario the object-oriented approach is widely accepted and used by the software professionals and researchers. The object-oriented approach is not only used in the programming language but also it is widely used in databases to design object-oriented databases. The object-oriented database was designed to meet the need of more complex applications that has huge database. It is the combination of database and object-oriented programming. The object-oriented database provides flexibility for handling the complex requirements without being limited the data types. The key part of the object-oriented database is it takes and stores the crisp information or data. It offers to access the data in a faster manner with respect to the traditional databases. The objectoriented database is very efficient and effective in such areas where the huge or massive amount of data about one data is required.In the real world applications the information is uncertain and vague. For handling that kinds of information the concept of fuzziness is introduce in the traditional relational database. But the relational database has store the redundant and ambiguous data. Therefore, software professionals and researchers have focused and moved towards the objet-oriented database to handle the complex objects and fuzzy data together. Therefore, a fuzzy objectoriented database is modeled to handle that kind of objects and fuzzy data.

\section{RELATED WORK}

As there is limited research is done on fuzzy object-oriented database; let us first describe the important work done previously related to the fuzzy object-oriented database. Sudhakar E. K. and Manimekalai M [1] have described the database of the heart disease where there is inaccuracy occurs, accurate information is provided for the users to help them a medical fuzzy database. Akinyokun et al [2] have proposed a Fuzzy Logic-based Expert System for the diagnosis of heart failure disease. Singh et. al. [3] have presented a conceptual model has been proposed for fuzzy object-oriented databases using unified modeling language. Chen [4] has combined the fuzzy SQL with object-oriented databases and establishes the structure of fuzzy object-oriented SQL for fuzzy query. Saxena and Kumar [5] have described an approach to send the information in the form of an object-oriented database on hand-held devices. Ephzibah and Sundarapandian [6] have proposed a system that finds a solution to diagnose the disease using some of the evolutionary computing techniques like genetic algorithm; fuzzy rule based learning and neural networks. Saxena and Kumar [7] have represented an objectoriented database through the well modeling language i.e. Unified Modeling Language (UML).Shukla et al [8] have surveyed different approaches regarding integration of fuzzy techniques in object oriented databases has been sketched, under numerous categories of conceptual data modeling, querying, indexing etc.Ma and Yan [9] have reviewed fuzzy conceptual data models proposed in the literature, where fuzzy ER/EER, IFO and UML data models are mainly discussed, and reviews the applications of fuzzy conceptual data models. Saxena et al [10] have proposed a Unified Modeling Language (UML) model for the Patient Registration System (PRS). The three dimensional Data Cubes are also designed for faster searching \& sorting of Patient Registration database. Yuko and Yasushi [11] have initially sought to model hospital-based cancer registration processes using the Unified Modeling Language (UML), to clarify functions. Methods: The object of this study was the cancer registry ofOsaka University Hospital. Yazici A. and Cinar [12] have introduced a conceptual data model by extending ExIFO to handle both complex and uncertain mainly, fuzzy objects and classes. Cross et al [13] have presented the initial research efforts to use ODMG-93 object data model standard as the basis for defining a fuzzy object data model since it is becoming a defacto standard and several object-oriented database vendors are currently releasing commercial products in compliance with this standard. Zadeh [14] has introduced 
the theory of fuzzy sets and fuzzy logic, two concepts that laid the foundation of possibility theory in 1977 . According to him "the theory of fuzzy sets is a step toward a rapprochement between the precision of classical mathematics and the pervasive imprecision of the real world... a rapprochement born of the incessant human quest for a better understanding of mental processes and cognition. $\mathrm{Ma}$ and Shen [15] introduced different levels of fuzziness into the class of UML and presented the corresponding graphical representations, with the result that UML class diagrams may model fuzzy information. Galindo et al [16] have presented a conceptual fuzzy model, so-called FuzzyEER, and a case tool (Fuzzy CASE), to help the database engineers to build the conceptual model for fuzzy databases which are represented in the book Fuzzy Databases: Modeling, Design and Implementation and this book has a real influence in the orientation of the databases coursesDistinguishing source code text. If Times Roman is not available, try the font named Computer Modern Roman. On a Macintosh, use the font named Times. Right margins should be justified, not ragged.

\section{UML MODELING FOR FUZZY OBJECT-ORIENTED DATABASE}

The static behavior of the patient diagnostic system (PDS) is represented here through the class diagram. There are several attributes and properties like aggregation, association and generalization are represented through the sub classes in the designed UML class diagram. In this work, a complete process of the patient registration and diagnostic system is explained using the UML class diagram that contains the several major classes like Patient, Registration Desk, MSW_Section, Doctor, Departments, Ward, Patient Admit, Patient Discharge,Pharmacy, Hospital_Info_System, Sample Collection/Report_Distibution, Bill Section and Patient History/Treatment represented in the Figure 1.
The Registration Desk class has multiple associations with the classes like (Patient, MSWand Bill Section). The Patient class has single association with Registration Desk class and multiple associations with the classes like Doctor,Patient History/Treatment,Pharmacy Patient Admit, Sample Collection/Report_Distibution and Patient_Disharge while the Patient Discharge class has multiple associations with the Bill Section class. The Doctor class has multiple associations with the Patient, Patient Admit, Patient History/Treatment, Hospital_info_System and Departments classes where the Departments class has single associations with the MSW class and generalized further in various departments like Neurology, Gynecology, Cardiology and Nephrology. All the departments have associated with the Wards class which is further generalized in Private and General Wards. According to the model i.e. (PDS) shown in the figure 1 the patients may arrive for registration and treatment whether they may be referred by the other doctors or they may come directly. If the concerned department and doctor are available the patients get the registration form from the registration desk and submit the filled registration form to the same desk. After registration a registration card and patient file is generated and also forwarded for screening the disease to the MSW section. The patients meet to the concerned doctor after the detailed screening of disease and if the doctor recommended for admit the patient in the ward then the patient will pay the admission charges as they preferred (General ward or Private ward) and the treatment of the patient is start. If the doctor feels that the condition and disease of the patient is not critical to be admit in the ward, the doctor prescribed the medicines and the patient takes the prescribed medicines from the pharmacy of the hospital and go home but the admitted patients may discharged after clearing all the treatments and tests bills when the doctor suggested that they are well.

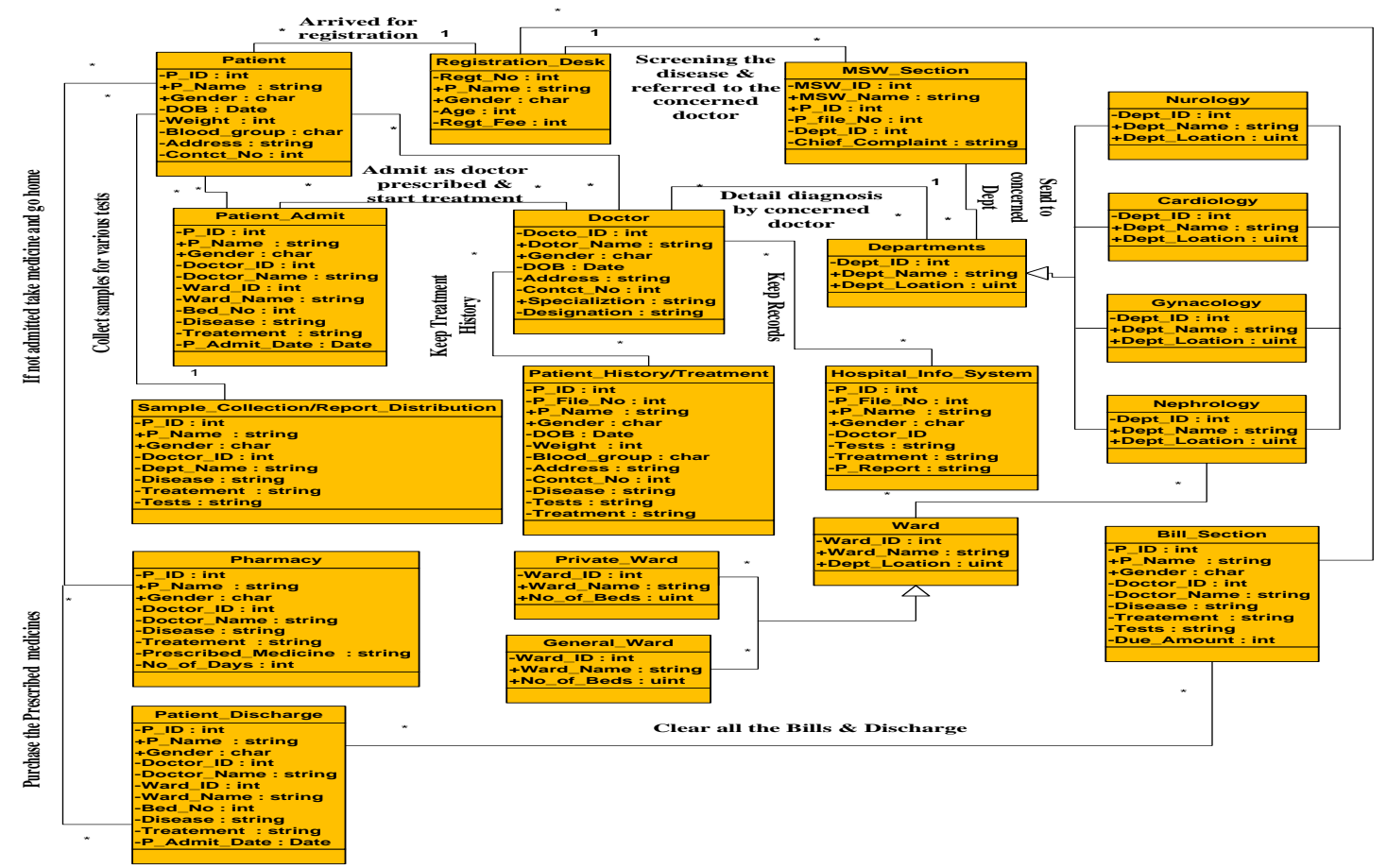

Fig 1: UML Class Diagram for Patient Diagnostic System

\subsection{UML Sequence Diagram}

As we have described the patient diagnostic system through the UML class diagram, a UML sequence diagram is designed for showing the static behavior of the above said work which is shown in the figure 2. From the figure it is shown that the patients have arrived to the hospital for their registration and 
their treatment. The sequence diagram shows the complete process of patient registration and diagnostic system. There are six major objects represented in the sequence diagram. The communication between the objects is shown by the solid arrow along with the communication messages. The dotted arrow shows the reply messages. The vertical line shows the life line of an object. The Patient has arrived to the Registration Desk in the hospital for the registration; after registration the MSW_Section screen the patient and referred to the concerned Department and concerned Doctor for the complete diagnosis. The Patient has admitted or not in the ward is depends on the Doctor's Prescription and starttreatment. As the admitted Patients well, the Doctor discharge the Patients after clearing all the bills.

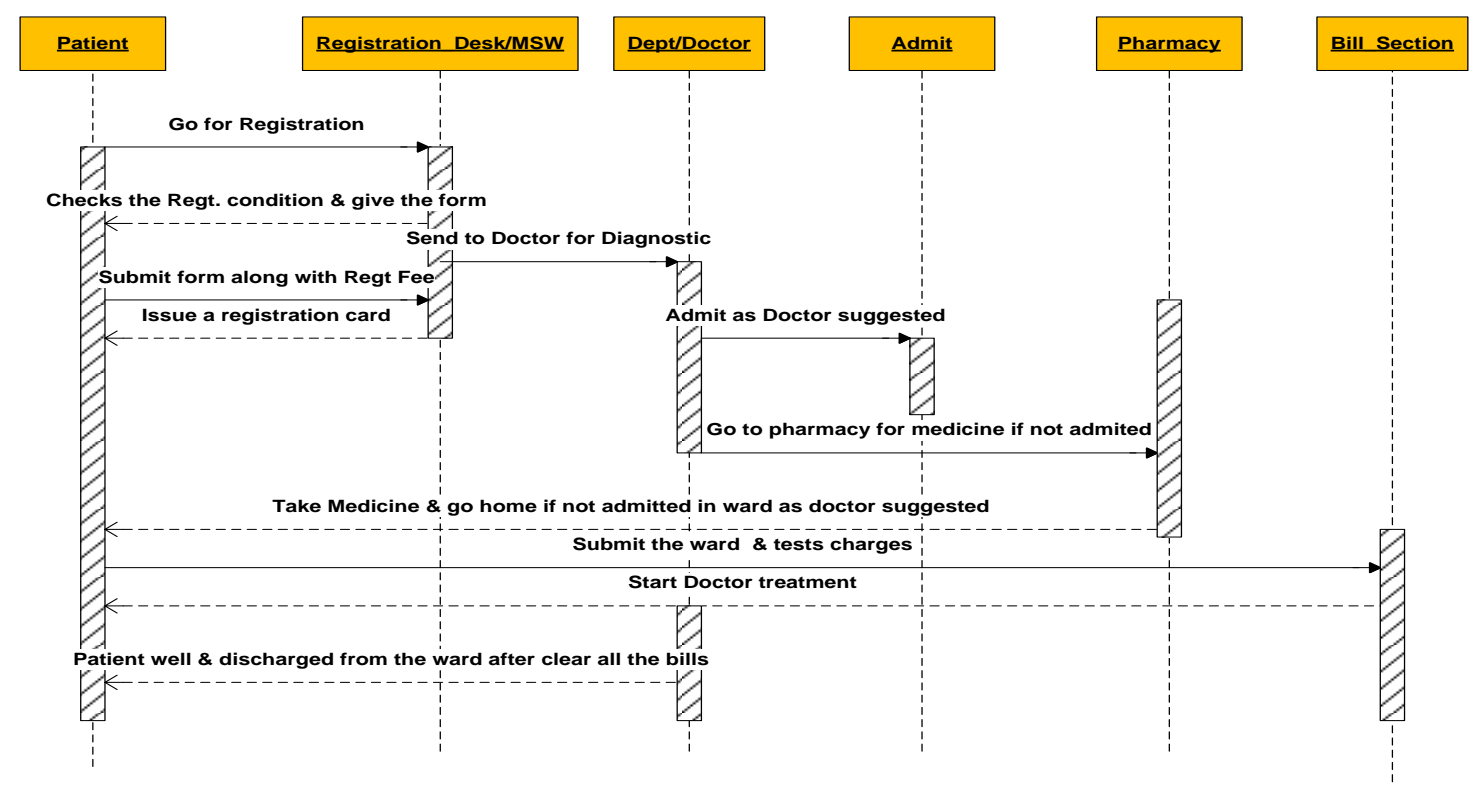

Fig 2: UML Sequence Diagram for Patient Diagnostic System

\section{EXPERIMENTAL RESULTS}

In this section a sample object-oriented database has been designed for the patient diagnostic system (PDS) for various diseases. The author has collected lots of real data from the hospital and designed a sample object-oriented database which is further extended to design a sample fuzzy objectoriented database and run some sample queries for both the designed databases to evaluate the performance of them.

\subsection{A Sample Object-Oriented Database for Patient Diagnostic System}

As the author has described an object-oriented database in the introduction section; the object-oriented database is designed by having the properties of a programming languages as well as the database also. An object-oriented database allows only the crisp data not the vague data. For designing the objectoriented database, the author has used the SQL server 2008. The designed database along with its tables is shown in Table 1 that has several major fields related to the patient which represent the brief look of all the records available in the master table i.e. Patient table. There are some sample queries are performed and corresponding results are given bellow: 
Table 1. Sample Object-Oriented Database for Patient DiagnosisSystem

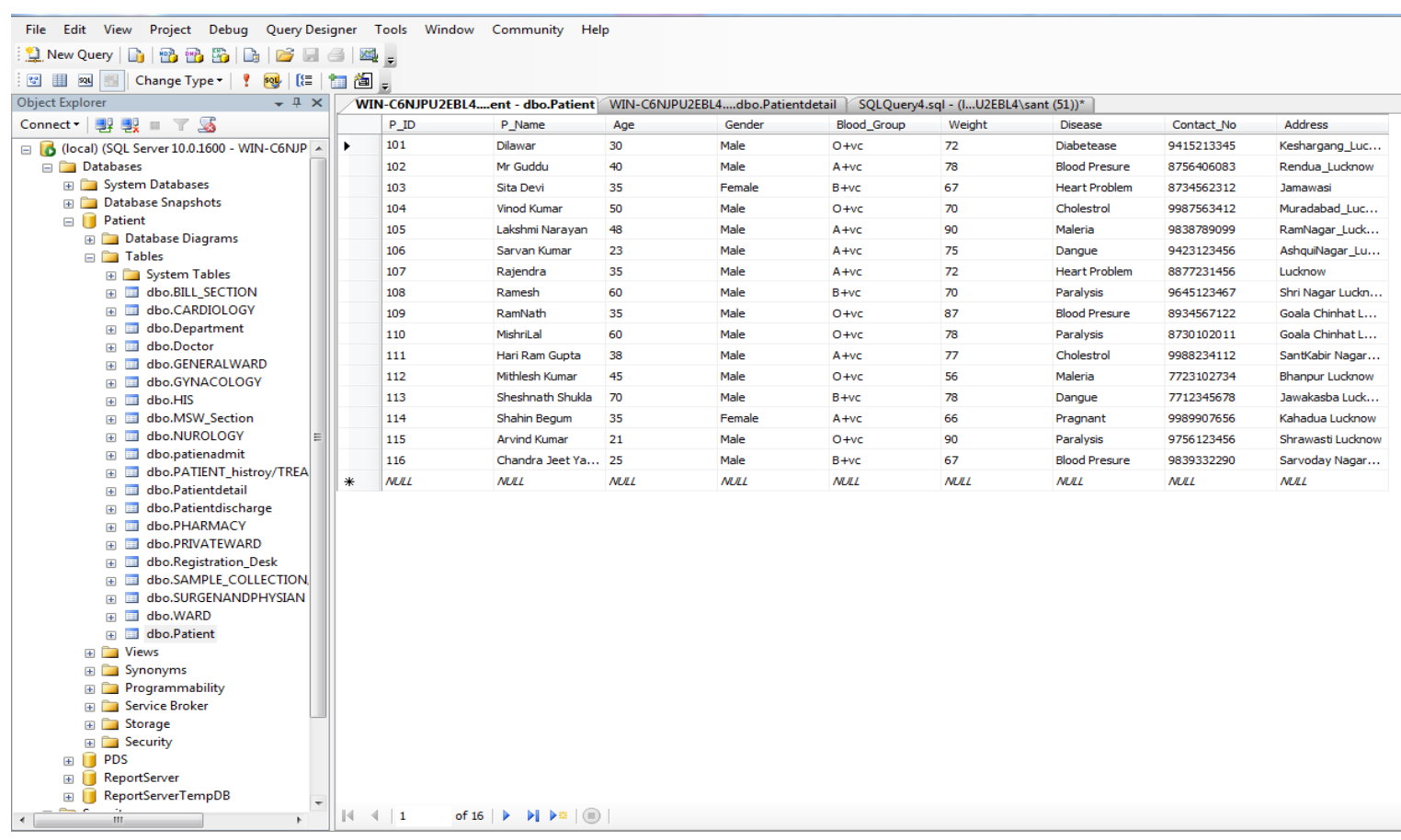

\section{Sample Query 1}

SELECT

[P_Name],[Gender]

FROM

[Patient].[dbo].[Patientdetail]

where Age $>25$ and Disease $=$ 'Blood Presure'

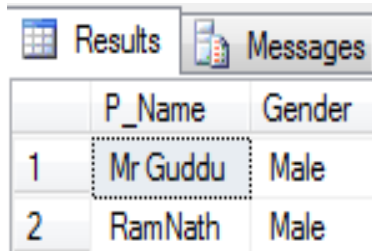

Sample Query 2:

SELECT [P_Name],[P_BloodGroup] FROM

[Patient].[dbo].[Patientdetail]where P_Age < 40;

\begin{tabular}{|l|l|l|l|}
\hline \multicolumn{1}{|c|}{ Results } & 湩 Messages & \\
\hline & P_Name & P_BloodGroup \\
\hline 1 & Dilawar & O+vc \\
\hline 2 & SitaDevi & B+vc \\
\hline 3 & SarvanKumar & A+vc \\
\hline 4 & Rajendra & A+vc \\
\hline 5 & RamNath & O+vc \\
\hline 6 & HariRamGupta & A+vc \\
\hline 7 & ShahinBegum & A+vc \\
\hline 8 & ArvindKumar & O+vc \\
\hline 9 & ChandraJeetYadav & B+vc \\
\hline
\end{tabular}

Sample Query 3:

SELECT [P_Name],[Gender],[Disease] FROM [Patient].[dbo].[Patient]where Age between 25 and 45

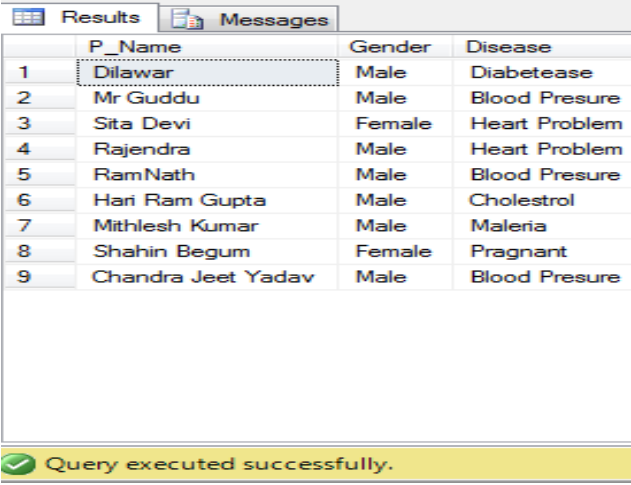

\subsection{Sample Fuzzy Object-Oriented}

Database for Patient Diagnostic System

In the field of medical sciences, there are so much vagueness and uncertainty found in the medical records. Therefore, it is very difficult to find a precise solution of a given problem on the basis of uncertain data. The process of diagnosis of any disease by using the fuzzy logic has several stages like uncertain values of symptoms of diseases, pathology test results i.e. given into range, diseases signs etc. Therefore, the

fuzzy object-oriented database gives the freedom to represent and instantiate that kind of uncertain and precise attributes values and strengthened the relations. According to the Galindo et al [18] the fuzzy object-oriented database model is an extension of graph-based object model that manage both certain (crisp) and vague (precise) values/information byusing fuzzy set theory and possibility theory. In this model a conceptual scheme is defined as a quintuple $\{\mathrm{C}, \mathrm{T}, \mathrm{A}, \mathrm{P}, \mathrm{N}\}$ : Where: 
1. C is a finite set of class names (crisp and fuzzy classes). Fuzzy classes collect objects, which can have a partial membership to the class.

2. $\quad \mathrm{T}$ is a finite set of type names (crisp and vague types). Vague types of elements denote sets of vague and imprecise values.

3. A is a set of attribute names. Attributes are simple when their domain is a type and are complex when their domain is a class. Moreover, both single-valued and multivalued attributes exist.

4. $\mathrm{P}$ is the property relation. $\mathrm{P}$ relates one class with its attribute names and both with the domain. This domain may be another class or type.

5. $\mathrm{H}$ is the inheritance relation. If the inheritance relation is fuzzy, then one label specifies the extent to which the instances of the subclass are also instances of the super class.

According to the Zadeh [15] the fuzzy logic is the logic behind approximate reasoning instead of exact reasoning. A fuzzy set is defined as: suppose $\mathrm{S}$ is a fuzzy set over auniverse of discourse $\mathrm{X}$ it is a finite or infinite interval within which the fuzzy set can take a value having a set of pairs:

$S=\left\{\mu_{\mathrm{s}}(\mathrm{x}) / \mathrm{x}: \mathrm{x} \in X, \mu_{\mathrm{s}}(\mathrm{x}) \in[0,1] \in \mathrm{R}\right\}$

Where the $\mu_{s}$ shows the membership degree/membership functionof the element $x$ to the fuzzy set $S$ and the universe of discourse $\mathrm{X}$ or the set of values being considered can be of two types:

1. Finite or discreet universe of discourse

$\mathrm{X}=\left\{x_{1}, x_{2}, \ldots \ldots, x_{n}\right\}$, where a fuzzy set $S$ can be represented by:

$$
S=\mu_{1} / x_{1}+\mu_{2} / x_{2}+\ldots+\mu_{n} / x_{n}
$$

2. Infinite universe of discourse, where a fuzzy set Aover X can be represented by:

$$
S=\int \mu \mathrm{s}(x) / x
$$

Here a fuzzy set $S$ \{Normal, Moderate, Critical, Very Critical $\}$ is defined for it input values like disease, signs, symptoms and pathology tests results. A membership function $\mu_{s}$ is defined for fuzzy input variables that are applied to determine the degree of truth. The set $\mathrm{S}=\left\{x_{1}, x_{2}, \ldots \ldots\right.$, $\left.x_{n}\right\}$ represent the input variables $\{$ signs, symptoms, pathology tests results $\}$. Therefore, the set is defined as:

$$
S=\left\{\mathrm{x}_{\mathrm{i}}, \mu_{\mathrm{s}}\left(\mathrm{x}_{\mathrm{i}}\right)\right\}
$$

Where $i=1,2,3,4, \ldots \ldots \ldots \ldots \ldots . n$.

Therefore, in membership function each variable described through the linguistic variables associated with the elements of the fuzzy set S i.e. Normal, Moderate, Critical, Very Critical. The output parameters are based on the linguistic variables and their associated fuzzy value range which is shown in the Table 2.
Table 2. Sample Table of Linguistic Variables along with their Fuzzy Values Range

\begin{tabular}{|l|l|}
\hline \multicolumn{1}{|c|}{ Linguistic Variables } & \multicolumn{1}{c|}{ Fuzzy Value Range } \\
\hline Normal & $\mathrm{x}=0.0-0.30$ \\
\hline Moderate & $\mathrm{x}=0.30-0.50$ \\
\hline Critical & $\mathrm{x}=0.50-0.70$ \\
\hline Very Critical & $\mathrm{x}=0.70-0.90$ \\
\hline
\end{tabular}

By incorporating the linguistic variables and their fuzzy values ranges, the fuzzy object-oriented database (FOOD) has been designed for the patient diagnostic system. There are several major fields like P_ID, P_Name, Disease, Linguistic Variable and Fuzzy Value are shown in the database Table 3 that shows the fuzzy range of the disease through which the

\begin{tabular}{|c|c|c|c|c|c|}
\hline & $P_{-} \mid \mathbb{D}$ & P_Name & Disease & Linguistic_Variables & Fuzzy_Value \\
\hline 1 & 101 & Dilawar & Dibbetease & Moderate & $0.30-0.50$ \\
\hline 2 & 102 & MrGuddu & Blood Presure & Moderate & $0.30-0.50$ \\
\hline 3 & 103 & Sita Devi & Heat Problem & Cintical & $0.50-0.70$ \\
\hline 4 & 104 & Vinod Kumar & Cholestrol & Moderate & $0.30-0.50$ \\
\hline 5 & 105 & Lakshmi Narayan & Malenia & Citical & $0.50-0.70$ \\
\hline 6 & 106 & Sarvan Kumar & Dangue & Very Citical & $0.70-0.90$ \\
\hline 7 & 107 & Rajendra & Heat Problem & Very Citical & $0.70-0.90$ \\
\hline 8 & 108 & Ramesh & Pardysis & Very Citical & $0.70-0.90$ \\
\hline 9 & 109 & RamNath & Blood Presure & Moderate & $0.30-0.50$ \\
\hline 10 & 110 & Mishrilal & Pardysis & Very Citical & $0.70-0.90$ \\
\hline 11 & 111 & Hari Ram Gupta & Cholestrol & Moderate & $0.30-0.50$ \\
\hline 12 & 112 & Mithlesh Kumar & Malenia & Citical & $0.50-0.70$ \\
\hline 13 & 113 & Sheshnath Shukla & Dangue & Very Citical & $0.70-0.90$ \\
\hline 14 & 114 & Shahin Begum & Pragnant & Cintical & $0.50-0.70$ \\
\hline 15 & 115 & Avind Kumar & Pardysis & Moderate & $0.30-0.50$ \\
\hline 16 & 116 & Chandra Jeet $Y_{a} . .$. & Blood Presure & Moderate & $0.30-0.50$ \\
\hline
\end{tabular}
doctor decided whether the patient is admitted or not.

Table 3. Sample FOOD for Patient Diagnostic System

\section{CONCLUDING REMARK}

From the above work, it is measured that the Unified Modeling Language i.e. UML can be easily applied on the object-oriented database. The present work show that an attempt is made for representing an object-oriented database for the domain of hospital and it is extended in the fuzzy object-oriented database which is also represented here. This work can be further extended for designing the 3D data cube that anyone can extract the information easy and faster manner about the patient details and other related details.

\section{ACKNOWLEDGMENTS}

Authors are grateful to Vice-Chancellor, Maharishi University of Information Technology Lucknow for providing the excellent facility in the computing lab of Maharishi university of Information Technology, Lucknow, India. Thanks are alsodue to University Grant Commission, India for support to the University. 


\section{REFERENCES}

[1] Sudhakar E. K. and Manimekalai M. 2015, "A Novel Methodology for Diagnosing the Heart Disease Using Fuzzy Database", International Journal of Research in Engineering and Technology Vol. 4, pp.84-89.

[2] Akinyokun O. C., Iwasokun G. B., Arekete S. A. and Samuel R. W. 2015, "Fuzzy logic-driven expert system for the diagnosis of heart failure disease", Artificial Intelligence Research, Vol. 4, No. 1, pp. 12-21.

[3] Singh S., Agarwal K., and Ahmad J. 2014, "Conceptual Modeling In Fuzzy Object oriented Databases Using Unified Modeling Language", International Journal of Latest Research in Science and Technology, Vol. 3, PP. 174-178.

[4] Rui-Yang Chen, 2013, "Fuzzy SQL Query in Fuzzy Object-Oriented Database", Journal of Data and Information Processing, Vol. 1, pp. 9-18.

[5] Saxena V. and Kumar S. 2012, "Object-Oriented Database Connectivity for Hand Held Devices",Journal of Software Engineering and Applications, USA, Vol. 5 No. 5, pp. 314-320.

[6] Ephzibah E.P., and Sundarapandian V. 2012, "A Neuro Fuzzy Expert System for Heart Disease Diagnosis" Computer Science \& Engineering: An International Journal (CSEIJ), Vol.2, No.1, pp. 17-23.

[7] Saxena V. and Kumar S. 2011, "Object-Oriented Database Representation through UML", International Journal on Computer Science and Engineering, Singapore, Vol. 3 No. 1, pp. 440-444.

[8] Shukla P. K., Darbari M., Singh V. K., and Tripathi S. P., 2011, "A Survey of Fuzzy Techniques in Object-
Oriented Databases", International Journal of Scientific and Engineering Research, Vol. 2, pp. 1-11.

[9] Ma Z. M. and Yan L. 2010, "A Literature Overview of Fuzzy Conceptual Data Modeling", Journal of Information Science and Engineering, Vol. 26, pp. 427439.

[10] Saxena V., Ansari A. G. and Kumar K. 2008, "Data Cube Representation of Patient Registration System through UML", International Journal of Computer and Network Security, VOL. 8, No.10, pp. 319-323.

[11] Yuko Ohno and Yasushi Matsumura, 2008, "Unified Modeling Language (UML) for Hospital-based Cancer Registration Processes", Asian Pacific journal of cancer prevention, Vol. 9, pp.789-796.

[12] Yazici A. andCinar A., 1998, "Conceptual Design of Fuzzy Object-Oriented Database", Second International Conference on Knowledge-Based Intelligent Electronic Systems, IEEE, pp. 299-305.

[13] Cross V., deCaluwe R. and Vangysehem N. 1997, "A Perspective from the Fuzzy Object Data Management Group," 6th IEEE, International Conference on Fuzzy Systems Barcelona, Spain, pp. 721-728.

[14] Zadeh L.A., 1965. Fuzzy Sets, Information and control, Vol. 8, pp. 338-353.

[15] Ma Z. M. and Shen D., "Modeling Fuzzy Information in the IF2O and Object-Oriented Data Models", Journal of Intelligent and Fuzzy Systems, Vol.17, pp. 597-612.

[16] Galindo J., Urrutia A. and Piattini M., "Fuzzy Databases: Modeling, Design and Implementation", Idea Group Publishing. 